\title{
ON COMPLETELY CONTINUOUS HANKEL MATRICES ${ }^{1}$
}

\section{PHILIP HARTMAN}

1. Main theorem. Let $x=\left(x_{0}, x_{1}, \cdots\right)$ be a sequence of complex numbers and let $|x|$ be the norm $|x|=\left(\sum\left|x_{n}\right|^{2}\right)^{1 / 2} \geqq 0$. Let an asterisk denote complex conjugation. If $|x|<\infty$ and $|y|<\infty$, let $[x, y]$ $=[y, x]$ denote the sum of the series $\sum x_{n} y_{n}$, that is, the "scalar" product of $y$ by $x^{*}$.

For a given $x$ with a finite norm $|x|$, let $x(t)$ denote a function of class $L^{2}(0,2 \pi)$ having the Fourier series

$$
x(t) \sim \sum_{n=0}^{\infty} x_{n} e^{i n t} .
$$

Functions which differ only on zero sets will be considered to be identical. Correspondingly, $x(t)$ will be called continuous if there exists a continuous periodic function $x(t)$ satisfying (1). Also, "bounded" and "sup" below mean "essentially bounded" and "ess sup."

With an $f=\left(f_{0}, f_{1}, \cdots\right)$ of finite norm, associate the infinite Hankel matrix

$$
H=H(f) \equiv\left(f_{n+m}\right), \text { where } n, m=0,1, \cdots .
$$

Then $[H x, y]$ is the bilinear form $\sum \sum f_{n+m} x_{n} y_{m}$. If $H$ is bounded in the sense of Hilbert, put

$$
\|H\|=\underset{|x|=1}{\text { l.u.b. }}|H x|=\underset{|x|=|y|=1}{\text { l.u.b. }}|[H x, y]| .
$$

Although it is not assumed that $H$ is hermitian (that is, that the components of $f$ are real-valued), it is easy to see that

$$
\|H\|=1 . u . b .|[H x, x]| \text { for }|x|=1 \text {. }
$$

It was pointed out by Toeplitz [6] that a sufficient condition for $H(f)$ to be bounded is that one of the two functions $F_{1}(t) \sim f_{0} / 2$ $+\sum f_{n} \cos n t, F_{2}(t) \sim i \sum f_{n-1} \sin n t$ be bounded on $(0,2 \pi)$. Recently, Nehari [5] gave the following extension of Toeplitz's sufficient condition to a necessary and sufficient condition for the boundedness of $H(f)$ :

Received by the editors April 10, 1958.

1 The preparation of this paper was sponsored by the Office of Naval Research. Reproduction in whole or in part is permitted for any purpose of the United States Government. 
(i) (Nehari) Let $f=\left(f_{0}, f_{1}, \cdots\right)$ have a finite norm. If $g=\left(0, g_{1}, g_{2}, \cdots\right)$ is of finite norm and

$$
F(t)=f(-t)+g(t) \sim \sum_{n=0}^{\infty} f_{n} e^{-i n t}+\sum_{n=1}^{\infty} g_{n} e^{i n t},
$$

then $\|H(f)\| \leqq \sup |F(t)|(\leqq \infty) . H(f)$ is bounded if and only if there exists a $g=\left(0, g_{1}, \cdots\right)$ of finite norm such that (4) is bounded; in which case, there exists a $g$ with the property that

$$
\|H(f)\|=\sup |F(t)| .
$$

It was pointed out in [4] that a sufficient condition for $H(f)$ to be completely continuous is that one of the two functions $F_{1}(t), F_{2}(t)$, above, be a continuous, periodic function. The main result of this paper is the following analogue of (i):

(ii) $H(f)$ is completely continuous if and only if there exists a $g=\left(0, g_{1}, \cdots\right)$ of finite norm such that $(4)$ is continuous.

2. Remarks. When $H=H(f)$ is completely continuous or whenever $H(f)$ has the property that $|[H x, x]|=\|H\|$ for some $x=x^{0}$ of unit norm, then a function (4) satisfying (5) also satisfies

$$
|F(t)| \equiv \text { const. }(=\|H\|)
$$

and $F(t) x(t)=\|H\| x^{*}(t)$ for $x=x^{0}$. This follows readily from the Toeplitz relation

$$
(2 \pi)^{-1} \int_{0}^{2 \pi} F(t) x^{2}(t) d t=[H x, x]
$$

for any (bounded) function (4) and any $x$ (of finite norm) and from the theorem of $\mathrm{F}$. and M. Riesz which states that if $x(t) \neq \equiv$, then $x(t)$ vanishes only on a zero set.

Furthermore, in this case, a function (4) satisfying (5), hence (6), is unique. For if $F^{j}(t)=f(-t)+g^{j}(t)$, where $j=1,2$, satisfy (4)-(5), then the same is true of $F(t)=F^{1}(t)+\lambda\left(g^{2}(t)-g^{1}(t)\right)$ for $0 \leqq \lambda \leqq 1$. But $F(t)$ satisfies $(6)$ for $0 \leqq \lambda \leqq 1$, so that $g^{2}(t)-g^{1}(t) \equiv 0$.

In the case of a completely continuous $H(f)$, it will remain undecided whether or not the unique $F(t)$ satisfying (4)-(6) is a continuous periodic function.

Nehari's proof of (i) depends on the following theorem of Carathéodory and Fejér [1]: Among all the functions $G(z)=a_{0}+a_{1} z+\cdots$, analytic and bounded on $|z|<1$ with the same $n+1$ initial coefficients $a_{0}, \cdots, a_{n}$, there exists a unique one for which

$$
M=1 . \text { u.b. }|G(z)| \text { on }|z|<1
$$


is the smallest; this unique $G(z)$ is of the form

$$
G(z)=M e^{i \phi} \prod_{k=1}^{n}\left(\alpha_{k}-z\right) /\left(1-\alpha_{k}^{*} z\right)
$$

where $\phi$ is a real constant and $\alpha_{1}, \cdots, \alpha_{n}$ are complex numbers satisfying $\left|\alpha_{k}\right|<1$. In particular, $G(z)$ is analytic on $|z| \leqq 1$ and $\left|G\left(e^{i t}\right)\right| \equiv M$.

Note that if $f=\left(f_{0}, f_{1}, \cdots\right)$ has only a finite number of components distinct from 0 , say $f_{j}=0$ if $j>n$, then the unique $F(t)$ satisfying (4)-(6) is given by $F(t)=e^{-i n t} G\left(e^{i t}\right)$, where $G(z)$ is the function belonging to the $n+1$ constants $\left(a_{0}, \cdots, a_{n}\right)=\left(f_{n}, f_{n-1}, \cdots, f_{0}\right)$.

A short proof of the Carathéodory-Fejér theorem is given by Nehari [5]; cf. Gronwall [3]. Curiously, Fischer [2] gives a proof of a related theorem of Carathéodory using finite sections of a Hankel matrix.

3. Two lemmas. The proof of (ii) will depend on the remarks of the last section and on either of the following two equivalent lemmas.

Lemma 1. Let $f=\left(f_{0}, f_{1}, \cdots\right)$ have a finite norm. $H(f)$ is bounded [completely continuous] if and only if there exists a sequence of vectors $f^{n}=\left(f_{0}^{n}, f_{1}^{n}, \cdots\right)$ each having only a finite number of nonvanishing components and such that $H_{n}=H\left(f^{n}\right)$ tends strongly [uniformly] to $H$ as $n \rightarrow \infty$.

In order to be able to state the second lemma, introduce the following notation: For a given vector $f=\left(f_{0}, f_{1}, \cdots\right)$ and number $r$, $0<r<1$, let $f^{r}=\left(f_{0}, r f_{1}, r^{2} f_{2}, \cdots\right)$ and $f^{r, n}=\left(f_{0}, r f_{1}, \cdots, r^{n} f_{n}, 0, \cdots\right)$.

Lemma 2. Let $f=\left(f_{0}, f_{1}, \cdots\right)$ have a finite norm. $H(f)$ is bounded [completely continuous] if and only if $H_{r}=H\left(f^{r}\right)$ tends strongly [uniformly] to $H(f)$ as $r \rightarrow 1-0$.

4. Proof of the lemmas. Note that $H_{n}$ in Lemma 1 is completely continuous, in particular, bounded. Consequently, a strong [uniform] limit of $H_{1}, H_{2}, \cdots$ is bounded [completely continuous]. This implies the "if" half of Lemma 1.

Note that for any pair of vectors $f^{1}$ and $f^{2}$, one has, by (i) and by $H\left(f^{1}-f^{2}\right)=H\left(f^{1}\right)-H\left(f^{2}\right)$,

$$
\left\|H\left(f^{1}\right)-H\left(f^{2}\right)\right\| \leqq \sup \left|f^{1}(t)-f^{2}(t)\right| \leqq \infty .
$$

Also, if $r$ is fixed on $0<r<1$, then

$$
\sup \left|f^{r}(t)-f^{r, n}(t)\right| \rightarrow 0
$$
as $n \rightarrow \infty$.

Thus $H\left(f^{r, n}\right)$ tends uniformly to $H\left(f^{r}\right)$ as $n \rightarrow \infty$. 
By the half of the Lemma 1 already verified, it follows that $H\left(f^{r}\right)$ is completely continuous for $0<r<1$. Correspondingly, the "if" half of Lemma 2 follows.

The "only if" part of Lemma 1 follows from (9), (10) and from the "only if" part of Lemma 2. Thus it only remains to prove the "only if" part of Lemma 2.

If $H=H(f)$ is bounded and $x$ is any vector of finite norm, then

$$
\left|\left(H-H_{r}\right) x\right|^{2}=\sum_{n=0}^{\infty}\left|\sum_{m=0}^{\infty} f_{n+m} x_{m}-r^{n} \sum_{m=0}^{\infty} f_{n+m} r^{m} x_{m}\right|^{2} .
$$

Thus $\left|\left(H-H_{r}\right) x\right|$ does not exceed

$$
\left|H\left(x-x^{r}\right)\right|+\left\{\sum_{n=0}^{\infty}\left(1-r^{n}\right)^{2}\left|\sum_{m=0}^{\infty} f_{n+m} r^{m} x_{m}\right|^{2}\right\}^{1 / 2} .
$$

Consequently,

$$
\left|\left(H-H_{r}\right) x\right| \leqq\left|H\left(x-x^{r}\right)\right|+(1-r)\left|H x^{r}\right| .
$$

Since $\left|x^{r}-x\right| \rightarrow 0$ as $r \rightarrow 1$, the boundedness of $H$ implies that $\left|H\left(x-x^{r}\right)\right| \rightarrow 0$ as $r \rightarrow 1$. Also, $(1-r)\left|H x^{r}\right| \leqq(1-r)|| H|||x| \rightarrow 0$ as $r \rightarrow 1$. Thus $H_{r}$ tends strongly to $H$ as $r \rightarrow 1-0$.

Suppose that $H=H(f)$ is completely continuous and suppose, if possible, that $H_{r}$ does not tend uniformly to $H$ as $r \rightarrow 1$. Then there exists a constant $c>0$, a sequence of $r$-values $r_{1}<r_{2}<\cdots$ and a sequence of unit vectors $x^{1}, x^{2}, \cdots$ such that $r_{n} \rightarrow 1$ as $n \rightarrow \infty$ and

$$
\left|\left(H-H_{r}\right) x^{m}\right| \geqq c>0 \quad \text { for } r=r_{n} .
$$

It can be supposed that $x=\lim x^{n}$, as $n \rightarrow \infty$, exists weakly. Then

$$
y^{n} \equiv x^{n}-x \rightarrow 0 \quad \text { weakly } \text { as } n \rightarrow \infty .
$$

By the triangular inequality, $\left|\left(H-H_{r}\right) x^{n}\right| \leqq\left|\left(H-H_{r}\right) y^{n}\right|$ $+\left|\left(H-H_{r}\right) x\right|$. As shown above, $\left|\left(H-H_{r}\right) x\right| \rightarrow 0$ as $r \rightarrow 1$. Thus (12) leads to a contradiction if it is shown that

$$
\left|\left(H-H_{r}\right) y^{n}\right| \rightarrow 0 \text { as } r=r_{n} \rightarrow 1 .
$$

The inequality (11), with $x$ replaced by $y^{n}$, gives

$$
\left|\left(H-H_{r}\right) y^{n}\right| \leqq\left|H y^{n}\right|+\left|H\left(y^{n}\right)^{r}\right|+(1-r)\left|H\left(y^{n}\right)^{r}\right|,
$$

where $\left(y^{n}\right)^{r}=\left(y_{0}^{n}, r y_{1}^{n}, \cdots\right)$ if $y^{n}=\left(y_{0}^{n}, y_{1}^{n}, \cdots\right)$. It is easy to see that (13) implies that $\left(y^{n}\right)^{r} \rightarrow 0$ weakly as $r=r_{n} \rightarrow 1$. Thus, from the complete continuity of $H$, it follows that $\left|H y^{n}\right| \rightarrow 0$ and $\left|H\left(y^{n}\right)^{r}\right| \rightarrow 0$ if $r=r_{n}$ and $n \rightarrow \infty$. This gives (14) and proves the two lemmas. 
5. Proof of (ii). The "if" part of (ii) is simple and is proved as is (b) in $\left[4\right.$, p. 365]. In fact, if there exists a $g=\left(0, g_{1}, \cdots\right)$ such that $F(t)$ in (4) is continuous, then there exist trigonometric polynomials $F^{n}(t)=f^{n}(-t)+g^{n}(t)$ which tend uniformly to $F(t)$ as $n \rightarrow \infty$. Since $H\left(f-f^{n}\right)=H(f)-H\left(f^{n}\right)$ and $\left\|H\left(f-f^{n}\right)\right\| \leqq \max \left|F(t)-F^{n}(t)\right| \rightarrow 0$ as $n \rightarrow \infty$, it follows from Lemma 1 that $H$ is completely continuous.

Conversely, suppose that $H=H(f)$ is completely continuous. Then, by Lemma 1 , there exist vectors $f^{1}, f^{2}, \cdots$, each having only a finite number of nonvanishing components and satisfying $\left\|H-H\left(f^{n}\right)\right\| \rightarrow 0$ as $n \rightarrow \infty$. On replacing the sequence by a subsequence, if necessary, it can be supposed that

$$
\left\|H_{n}-H_{n+1}\right\|<2^{-n}, \text { where } H_{n}=H\left(f^{n}\right),
$$

for $n=1,2, \cdots$.

Since $f^{n}-f^{n+1}$ has only a finite number of nonvanishing components, there exists a unique vector $g^{n}=\left(0, g_{1}^{n}, \cdots\right)$ such that $g^{n}(t)$ is continuous and

$$
\left|f^{n+1}(-t)-f^{n}(-t)+g^{n}(t)\right| \equiv\left\|H_{n}-H_{n+1}\right\| ;
$$

cf. §2. By (15) and (16), it follows that

$$
F(t)=\lim _{n \rightarrow \infty}\left[f^{n+1}(-t)+\sum_{k=1}^{n} g^{k}(t)\right]
$$

exists uniformly for all $t$ and, hence, is a continuous, periodic function.

It is clear that $|f| \leqq\|H\|$. In fact, the first component of $H f^{*}$ is $|f|^{2}$, so that $|f|^{2} \leqq\left|H f^{*}\right| \leqq|| H|||f|$. Hence, $\left\|H-H_{n}\right\| \rightarrow 0$ implies that $\left|f-f^{n}\right| \rightarrow 0$ as $n \rightarrow \infty$. This shows that (17) is of the type (4) and completes the proof of (ii).

\section{REFERENCES}

1. C. Carathéodory and L. Fejér, Ueber den Zusammenhang der Extremen von harmonischen Funktionen mit ihren Koeffizienten und über den Picard-Landauschen Satz, Rendiconti del Circolo Matematico di Palermo vol. 32 (1911) pp. 218-239.

2. E. Fischer, Ueber das Carathéodorysche Problem, Potenzreihen mit positiven reellen Teil betreffen, ibid., pp. 240-256.

3. T. H. Gronwall, On the maximum modulus of an analytic function, Ann. of Math. vol. 16 (1914) pp. 77-81.

4. P. Hartman and A. Wintner, On the spectra of Toeplitz matrices, Amer. J. Math. vol. 72 (1950) pp. 359-366.

5. Z. Nehari, On bounded bilinear forms, Ann. of Math. vol. 65 (1957) pp. 153-162.

6. O. Toeplitz, Zur Theorie der quadratischen Formen von unendlich vielen Veränderlichen, Göttingen Nachrichten, 1910, pp. 489-506.

University of California at Los Angeles and

The Johns Hopkins University 